\section{Applied Research and Smart Technology}

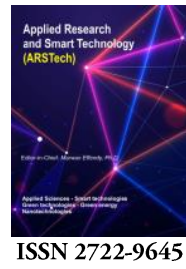

Journal homepage: www.journals2.ums.ac.id/index.php/arstech

Research Article

\title{
Wearable upper limb motion assist robot for eating activity
}

\author{
Uzair Kashtwari ${ }^{1}$, Norsinnira Zainul Azlan ${ }^{2, *}$, Ifrah Shahdad ${ }^{3}$ \\ ${ }^{1,2,3}$ Department of Mechanical Engineering, Kulliyyah of Engineering, International Islamic University Malaysia, \\ Jalan Gombak, 53100. Kuala Lumpur, Malaysia.
}

${ }^{*}$ Corresponding author: $\underline{\text { sinnira@iium.edu.my }}$

Permalink (DOI): https://doi.org/10.23917/arstech.v1i1.28

\begin{tabular}{|c|c|}
\hline ARTICLE INFO & ABSTRACT \\
\hline $\begin{array}{l}\text { Keywords: } \\
\text { Activities of daily living } \\
\text { Degree of freedom } \\
\text { Motion assist robots } \\
\text { Upper limb rehabilitation robot } \\
\text { Task-specific } \\
\text { Wearable exoskeleton }\end{array}$ & $\begin{array}{l}\text { Many people all around the world are suffering from various types of disabilities } \\
\text { and need to depend on others to perform activities of daily living. One of the } \\
\text { essential daily living activities is eating. The disabled people should be able to eat } \\
\text { their food independently at any time and place, without relying on the caregivers. } \\
\text { This paper presents the development of a new wearable upper limb motion assist } \\
\text { robot for helping the disabled to eat by themselves. The motion assists robot } \\
\text { consists of two degrees of freedom (DOF) movement, focusing on the two most } \\
\text { crucial upper limb movements in eating activity, which is the elbow } \\
\text { flexion/extension and forearm pronation/supination. A light-weight material was } \\
\text { used for the fabrication of the wearable motion assist robot, and Arduino was } \\
\text { utilized as the microcontroller. The originality of the study was in terms of the } \\
\text { design, operational sequence setting, and kinematic analysis of the wearable } \\
\text { upper limb motion assist robot that was explicitly focusing on eating activity. } \\
\text { The resulted prototype was portable, compact, light in weight, simple and low } \\
\text { cost. The experimental results have proven that the proposed wearable upper } \\
\text { limb motion assist robot for eating activity was successful in helping the users to } \\
\text { perform the main upper extremity motions in eating. The success rate of the } \\
\text { proposed system was } 80 \% \text {, and it took } 6 \text { seconds for the system to complete one } \\
\text { feeding cycle. }\end{array}$ \\
\hline
\end{tabular}

\section{INTRODUCTION}

Many people who losses his/her upper limb capability in performing daily living activities increases with the growing number of disabled due to ageing, accidents, and various types of illness. Malaysian is expected to become an ageing nation by 2030 , where at least $15 \%$ of its population will be above 60 years old. The number of stroke patients in Malaysia has increased tremendously over the past few years, in which an average of 90 stroke patients was admitted to the public hospitals every day in 2016 [1]. It was also reported that many athletes [2] and female metal manufacturing workers were suffering from chronic wrist pain [3]. These conditions left the patients 
with various contractual and deficiencies in their upper extremity, thus leading to frustration and disappointments since they were unable to perform self-care routines and daily tasks by themselves and need to depend heavily on the caregivers. One of the essential activities in daily living is eating. Most people consume at least twice a day, and this can be troublesome for both disabled people and caregivers. The disabled people should be able to eat independently at any time and place, at their convenience [4]-[5]. One of the methods to alleviate this problem is to have a wearable upper limb motion assist robot that can help while at the same time, trains the disabled to eat independently.

Numerous researches on wearable motions assist robots have been carried out across the world to cater to people with various needs [6]-[10]. Imoto et al. have developed an exercise assist robot for forwarding and backward movements. Many parameters, including the displacement of the centre of gravity and centre of pressure, angular displacements of the hips and knee joints, and maximum muscle activities of biceps femoris while the subjects were walking have been reduced with the utilization of the motion assist robot [5]. A walking assist robot and its biologically-inspired control strategy have been developed by Zhang et al. to integrate the hip central pattern generator control, a knee hierarchical impedance control, and a hip-knee linkage control to produce natural hip-knee motions [8]. Sun et al. developed an intelligent pneumatic artificial muscle actuated lower limb motion assist robot. The movement of the exoskeleton-type motion assists robot was driven by electromyography (EMG) signals that were proportional to the force measured between the robot and its human user [9]. A wearable hip assist robot with the potential to increase the stability of the elderly's trunk while walking has been designed by Lee et al. The motion assists robot decreased the muscle effort and enhanced the gait function of an ageing person [10].

Two types of assist robot could facilitate the disabled people for eating independently, which were the meal assist robot [11]-[21] or also known as feeding robot, and wearable exoskeleton-type robot [22]-[31] that could assist them to realize healthy arm movements in performing eating actions. Some examples of the meal assistance robot were the one that was developed based on Willow Garage PR2, a general-purpose mobile manipulator for the disabled with severe motor impairments. The robotic arm was equipped with a user interface, visual guidance, and safety features. It was able to transfer food to the disabled's mouth, requiring minimal head movement [12]. Guo et al. designed a 4 degrees of freedom (DOF) robot arm for feeding assistive function with four active joints. The forward kinematic analysis and trajectory planning of the assistive feeding robot has been successfully simulated [13]. The meal assistance robot developed by Nozaki et al. was made using 3D printers and focuses on two main motions for feeding, which were the translational and rotational motions for scooping and bringing the food to the mouth. The robot adopted the manual polar coordinate-based scheme so that the users could manoeuvre the robot intuitively [14]. A robotic arm equipped with a spoon for meal support has been developed by Yosioka et al. to scoop semi-liquid food automatically. The shape of the container needs to be known a priori, and the amount of scooped food and scooping success rate have been increased by the proposed scraping motion strategy and controlled spoon motion [15]. An automatic omnidirectional moving plate and orthogonal coordinate have been introduced by Takonari et al. to allow the option of eating multiple dishes from more than one container, where the food did not need to be organized before being consumed [16].

The trajectory for eating motion could be learned by the meal assistance robot using kinesthetics learning, where the robot learned from a teacher or operator demonstration along a guided path. In this learning method, the algorithm calculated the cost function of a robot's and caretaker's knowledge of the constrained feeding task [17]. A meal assistance robot could be driven by the user's head movement [18], the blink of eyes [19], electroencephalography (EEG) [20], and EMG signals [21]. In Tomimoto et al. 's design, a Kinect sensor was used to detect the patient's head movement, and then, the information was transferred to a Rasberry $\mathrm{Pi}$, which in turn instructs the orthogonal structured robot to move according to the head motion [18]. Perera et al. implemented the user's intention detection method based on Steady-State Visual Evoked Potentials (SSVEP) in an EEG controlled meal assistance robot. The users were able to choose any solid food that they would like to have. The robot was also equipped with a camera to detect the user's mouth position and recognize the opening or closing of the mouth [20]. Even though many meal assistance robots have been developed, most of these robots are bulky and less portable. Since it is a separate robotic arm unit, it is less appealing in terms of appearance and may cause the users to feel unsafe while eating. The robots also are not wearable; thus, they do not facilitate in training the disabled to regain their upper limb function to eat independently.

Many types of wearable exoskeleton for assisting for the upper limb motion while also rehabilitating the upper extremity have been developed [22]-[31]. This type of movement assist robot was capable of providing the training to help the disabled people to recover their original upper limb functionalities. The robot could be made of a low or high number of degrees of freedom (DOF) to accomplish the specific desired tasks. Inoue and Noritsugu designed a single actuator driven wearable upper limb motion assist robot, focusing on the shoulder 
and elbow movements in transferring loads. The wearable upper-limb motion assists robot structure was built based on the parallel link mechanism, and it was attached to the motor through a trapezoidal feed screw [23]. An exoskeleton robot for 3 DOF wearable upper-limb motion assist robot, focusing on the shoulder and Kiguchi et al. have developed elbow flexion/extension movements. The exoskeleton was equipped with an obstacle avoidance algorithm and natural hand trajectory motion [24]. Kiguchi et al. also developed a 7 DOF power-assist exoskeleton robot to support the elderly's or physically weak person's upper limb in carrying out daily activities. EMG signals drove the robot, and the impedance parameters in the robot were updated automatically in real-time in proportion to the user's arm posture and muscle activities [25]. The number of DOF of an upper limb motion assist robot could be kept high while reducing the structure's complexity by introducing underactuated joints as in [26], where the move help robot had 3 DOF planar joints and 1 DOF passive joint. The experimental tests in assisting human's upper limb movement along straight, square, and free curve paths have shown that the proposed motion help robot was suitable for rehabilitation and facilitating impaired arms in completing the assigned job [26]. Other wearable motions assist robots included the work by Natividad et al. on a modular, pneumatic, soft robotic exoskeleton for the shoulder abduction exercises. The surface EMG signals measured have shown that the proposed wearable exoskeleton has successfully assisted the limbs and reduced muscle activation [27]. The planarjoint structured upper limb motion assist device for hemiplegic patients proposed by $\mathrm{Hu}$ et al. was able to facilitate the patients to transfer things, eat, drink, and turn book pages. The robot could be driven by the user's voice and operate in three auxiliary modes, which are single DOF tasks, plane-motion activities, and threedimensional action facilitations. It enhanced the capability of the patients to perform daily routine by themselves while helping them to recover their upper limb's natural movement and functionality [28]. Liao et al. removed the need to have a predefined trajectory in the upper limb assistance device in performing Activities of Daily Living (ADL) by using the Microsoft Kinect 2 system to detect the human motion and drive the assistive device accordingly. Several human arm movements, while conducting daily activities, could be realised based on human intention without the need for predefined trajectories [29]. Passive and active control strategies based on the integral backstepping controller with Time Delay Estimation (BITDE) have been proposed for a 7-DOF exoskeleton robot in providing physical assistance and rehabilitation to the patients. The controller compensated for the uncertainties in the human arm and robot structure and external disturbances in tracking the desired trajectory [30]. Estimation of Desired Motion Intention (DMI) and model reference-based adaptive impedance control algorithm have been implemented on a 7 DOF upper limb assist exoskeleton. The control scheme overcomes the robot and human model uncertainties problems, and issues regarding local minima, selection of suitable parameters, slow convergence of adaptation law and overfitting in neural network-based estimation [31]. Even though positive results have been reported in these works, most of the previous wearable exoskeleton for assisting and rehabilitating the upper limb motion was developed for general movements or daily living activities. It did not specifically focus on eating exercise. Most of the previous upper limb motion assist robots were also not portable, and this may be inconvenient for the disabled, especially for those with low mobility levels. Many of the existing motion assist robots were too complicated, bulky, heavy, and expensive.

Therefore, this paper presents the development of a wearable exoskeleton type upper limb motion assist robot, concentrating on eating function. It could help the disabled people to eat alone while training their upper limb to regain the original motor functions. The proposed wearable upper limb motion assist robot is simple, lightweight, portable, and low cost. The paper is organised as follows: the mechanical and electrical design of the proposed movement assist robot would be presented, followed by the robot's operational sequence. Then, the kinematics analysis of the proposed motion assist robot would be described, and the experimental tests, user feedback, and findings would be discussed. Finally, the conclusion is drawn at the end of the paper.

\section{SYSTEM DESIGN}

In the proposed system design, a plate or bowl of food was assumed to be placed on the table. The proposed motion assist robot would facilitate the patients to scoop the food from the plate or bowl to his / her mouth using a spoon repetitively. The system needed to provide two simultaneous functionalities which were 1) actualize the upper limb movements in terms of the complete spatial configuration of the limb in realizing human motion while eating, 2) able to generate sufficient torque to move the disabled arms through the points of attachment.

The main upper limb joints to accomplish human eating motion under the most straightforward and lowest cost conditions while able to achieve the two functions above have been identified as the joints for the elbow flexion and extension motions, and forearm pronation and supination movements. The elbow motion brought the food from the bowl to the mouth and return. While, the forearm functioned to scoop the food from the bowl, and also put it into the mouth. Therefore, the proposed upper limb motion assist for an eating function was made of two links, which were the upper arm link and the lower arm 
link, as shown in the mechanical drawing in Figure 1. Both of the sizes of the links were made close to the human arm's length. These were attached to the arms holders that are made of $3 \mathrm{D}$ printed materials. The arm holders were equipped with slots where Velcro straps could be inserted to tighten or loosen the wearable motion assist robot to a human arm. Velcro straps were chosen in this design since they were flexible and able to accommodate various arm sizes.

Two motors were incorporated in the design, with one motor mounted at the elbow joint and one motor installed for the forearm pronation and supination motions at the end of the distal upper limb area, as shown in Figure 1. The motors were directly fixed to the linkages to achieve a compact design. The motor for forearm pronation and supination was placed at the end of the distal upper limb instead of positioning it at the elbow joint to avoid any mechanical interference and so that the robot was able to move more efficiently in realising the eating movement. The centre of rotation of the elbow and forearm motor shafts should be in line with the centre of rotation of the person's elbow and forearm, respectively, to provide the proper eating motions and prevent any pain during the motion assist. Therefore, a careful measure needed to be taken while putting on the wearable motion help robot for the patients. The length of the rods that connect the links and the arms holders also needed to be chosen carefully to ensure that the human arm can fit adequately in the motion assist robot. A round knob was attached to the shaft of the forearm motor, and the disabled can grasp the knob during the eating process. It increased their comfort while the forearm scoops the food from the bowl and puts it into the mouth. The clockwise or anticlockwise motion of the forearm motor drives the round knob and eventually turns the patient's forearm to pronate or supinate. Besides the spoon, another detachable eating device such as the fork could also be attached to the base of the knob.

DC servomotor has been chosen as the actuating device in the proposed system design due to its ability to attain the desired angle of rotation according to the signal sent to the motor. The output shaft of the servomotor could be moved and changed to a specific angular position easily based on the provided coded signal. As such, this type of motor was beneficial when a precise rotation is required. A DC servomotor with higher torque was used for the elbow extension and flexion since it needs to support a higher payload. In contrast, a lower torque DC servomotor was used for the arm pronation and supination. The length and location of the centre of gravity of the human arm have been taken into consideration in choosing the DC servomotor to actuate the elbow joint. The Regression equation for calculation of the arm segment lengths can be written as

$$
L_{\text {forearm }}=-14.54-0.22 G+0.305 H \text {, }
$$

where $G$ is the mass of the whole body, and $H$ is the body height.

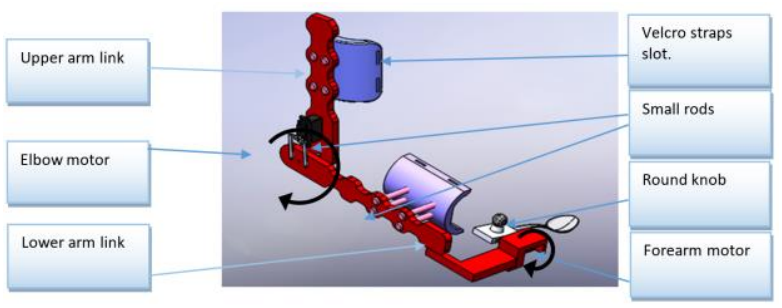

(a)

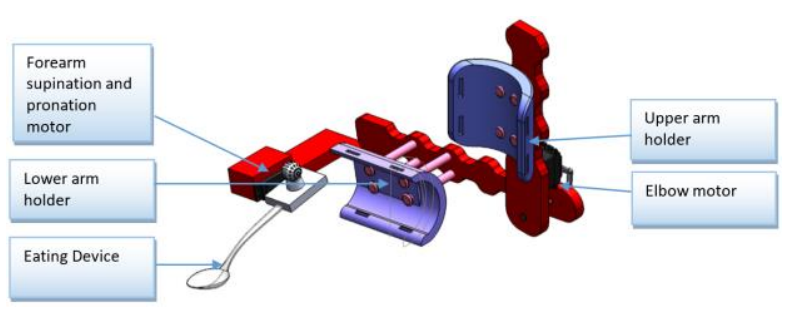

(b)

Figure 1. (a) Dorsal and (b) ventral views of the proposed wearable upper limb motion assist for eating.

The location of the centre of gravity along the longitudinal axis, measured from the distal end to the proximal end segment of the arm can be obtained by

$$
L_{C G}=42.74 \% \times L_{\text {forearm }},
$$

The regression equations for calculating the arm segment's mass is given by

$$
M_{\text {forearm }}=(0.165+0.0139 G),
$$

The torque to move the arm and motion assist robot for elbow flexion and extension can be calculated as

$T=\left(M_{\text {forearm }}+M_{\text {mechanism }}\right) \frac{\left(L_{C G}+L_{C \text { Gmechanism }}\right)}{2} g n_{s}$,

where $M_{\text {mechanism }}$ is the mass of the robotic mechanism obtained from the weighing machine, $L_{C G m e c h a n i s m}$ is the centre of gravity of the mechanism from the Solid works drawing software, $\mathrm{g}$ is the gravitational acceleration and $n_{s}$ is the safety factor chosen as 3 .

Monster Torque Brushless Servo (XQ-S5650D) and RC Servo Motor (Metal Gear) have been chosen for the elbow motor and forearm motors, respectively. Even though the Monster Torque Brushless Servo is quite costly, which is RM 700, it has been selected since it fulfils the torque requirement, is compact and has a high power to weight ratio, which is essential consideration factors in the prototype development. The motor specifications are summarized in Table 1. 
Table 1. Servo motor specifications.

\begin{tabular}{lcc}
\hline & $\begin{array}{c}\text { Monster Torque } \\
\text { Brushless Servo } \\
(\text { XQ-S5650D) }\end{array}$ & $\begin{array}{c}\text { RC Servo Motor } \\
\text { (Metal Gear) }\end{array}$ \\
\hline Speed & 0.18 seconds $/ 60^{\circ}$ & 0.14 seconds $/ 60^{\circ}$ \\
Torque & $60 \mathrm{~kg} . \mathrm{cm}$ & $1.66 \mathrm{Nm}$ \\
Voltage & $8.5-11 \mathrm{VDC}$ & $6 \mathrm{VDC}$ \\
Size & $60 \times 30 \times 60 \mathrm{~mm}$ & $40.7 \times 20.5 \times 39.5 \mathrm{~mm}$ \\
Weight & $177.5 \mathrm{~g}$ & $60 \mathrm{~g}$ \\
Rotation angle & $210 \mathrm{degrees}$ & 180 degrees \\
\hline
\end{tabular}

Arduino Uno R3 microcontroller board based on the ATmega328 has been selected as the main microcontroller. It allowed higher transfer rates and converts numerical values to pulse width modulation (PWM) signals for the motor controller. Arduino Uno R3 has been chosen primarily due to the availability of its open-source software, the ability to be programmed quickly and directly connected to the virtual COM port. The two servomotors were connected to a motor driver, which was attached to the Arduino board and a battery source. An 11.1V lithium-ion battery was used to power up the system since it was rechargeable and provides high energy storage to weight ratio. Potentiometers were used for measuring the movement of the robot. An emergency button was incorporated in the system to stop the robot's motion, hence, avoids any injuries or discomforts due to accidents or technical mishaps. The full-motion assist robot for an eating function is illustrated as in Figure 2, and the total cost of the robot is RM 950. At this stage of the study, the links were made of wood considering the total prototype cost, weight, and fabrication simplicity for proof of concept. However, later, the material can be upgraded for a more durable design and more extended life cycle operation.

\section{OPERATIONAL SEQUENCE}

Figure 3 presents the operational flow of the upper limb motion assist robot. After the disabled or patients wear the movement assist robot, the process began with the extension of the elbow by the elbow motor until the desired extension angle was reached, which places the hand close to the bowl. Then, the forearm motor actualised the forearm pronation and supination movements to scoop the food under the pre-set angular displacements. Next, the elbow motor flexed the elbow joint and brought the menu to the mouth. Then, the elbow joint extended again to scoop the food, and the process was repeated from the beginning for several cycles up to the stop button was pressed. The whole process was also put into a halt in case the emergency button is pressed.

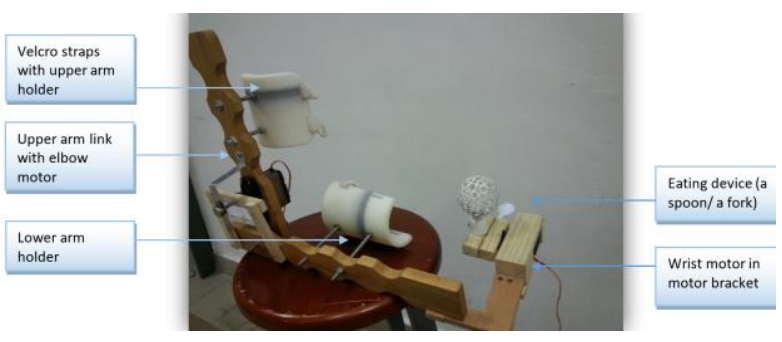

(a)

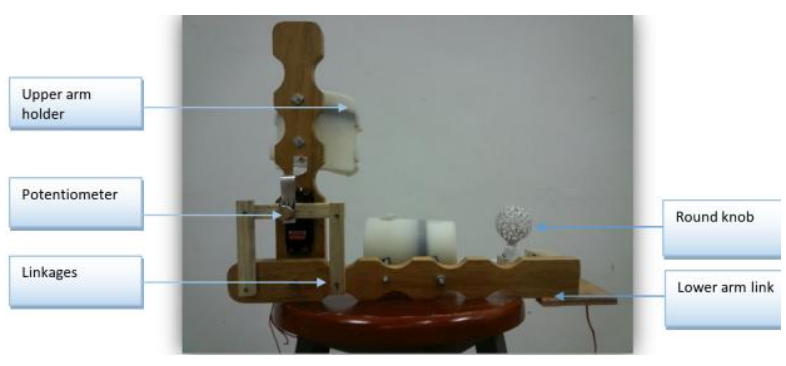

(b)

Figure 2. (a) Isometric and (b) side view of the upper limb motion assist robot for eating.

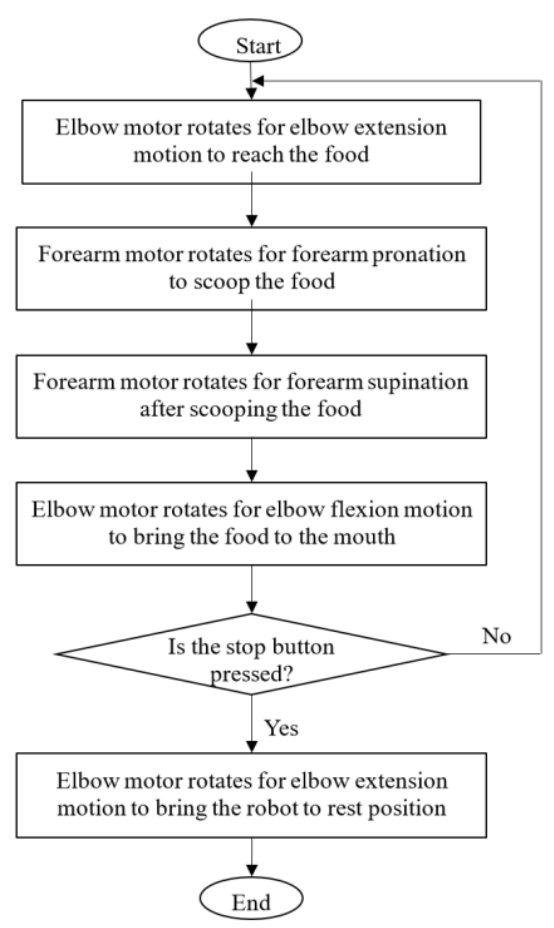

Figure 3. The operational flow of the wearable upper limb motion assists the robot in eating activity.

\section{KINEMATICS ANALYSIS}

Forward and inverse kinematics analyses have been conducted on the proposed wearable upper limb motion assist robot based on Denavit Hatenberg (DH) representation. DH is a general step by step method to obtain the total transformation matrix of the robot by combining the array of each frame. The reference frame 
for each joint of the proposed robot has been assigned as in Figure 4. The DH parameters have been obtained as in Table 2.

Table 2. DH parameters for the proposed wearable upper limb motion assist robot.

\begin{tabular}{ccccc}
\hline Link & $\theta$ & $d(\mathrm{~m})$ & $a(\mathrm{~m})$ & $\alpha$ \\
\hline 1 & $\theta_{1}$ & $l_{2}$ & $l_{1}$ & $-90^{\circ}$ \\
2 & $\theta_{2}$ & 0 & 0 & $0^{\circ}$ \\
\hline
\end{tabular}

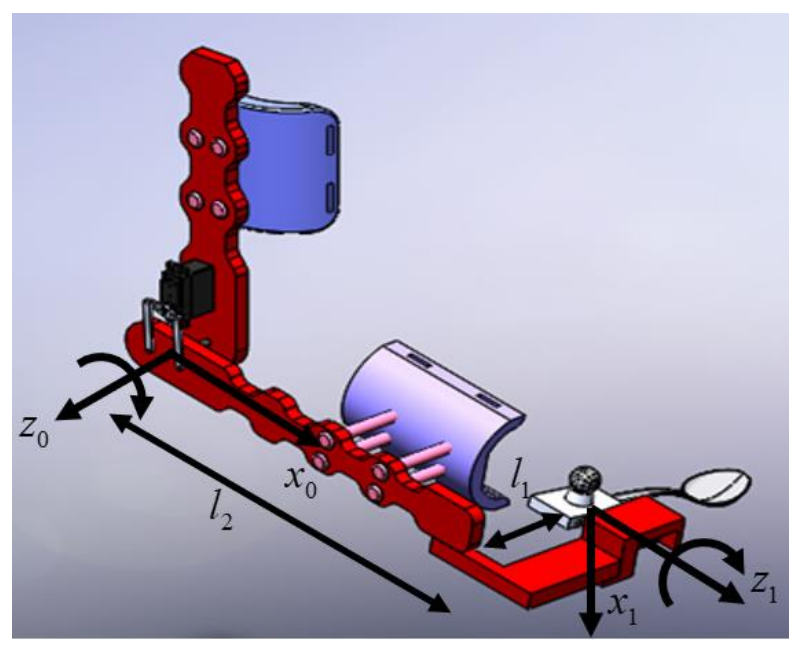

Figure 4. Reference frame assignment for $\mathrm{DH}$ representation

In forward kinematics analysis, the angular displacement of each joint is known, and the position and orientation of the end effector are calculated from the $\mathrm{DH}$ representation. From Table 2, the forward kinematics of the anthropomorphic robotic arm, $A_{\mathrm{n}}$ for $n^{\text {th }}$ link, can be obtained as follow

$A_{1}=\left[\begin{array}{cccc}\cos \theta_{1} & 0 & -\sin \theta_{1} & l_{2} \cos \theta_{1} \\ \sin \theta_{1} & 0 & \cos \theta_{1} & l_{2} \sin \theta_{1} \\ 0 & -1 & 0 & l_{1} \\ 0 & 0 & 0 & 1\end{array}\right]$

$A_{2}=\left[\begin{array}{cccc}\cos \theta_{2} & -\sin \theta_{2} & 0 & 0 \\ \sin \theta_{2} & \cos \theta_{2} & 0 & 0 \\ 0 & 0 & 1 & 0 \\ 0 & 0 & 0 & 1\end{array}\right]$

Multiplying $A_{1}$ and $A_{2}$ gives the total transformation matrix of the robot, $A_{1} A_{2}$ where

$A_{1} A_{2}=\left[\begin{array}{cccc}\cos \theta_{1} \cos \theta_{2} & -\cos \theta_{1} \sin \theta_{2} & -\sin \theta_{1} & l_{2} \cos \theta_{1} \\ \sin \theta_{1} \cos \theta_{2} & -\sin \theta_{1} \sin \theta_{2} & \cos \theta_{1} & l_{2} \sin \theta_{1} \\ -\sin \theta_{2} & -\cos \theta_{2} & 0 & l_{1} \\ 0 & 0 & 0 & 1\end{array}\right]$
The whole transformation matrix of the wearable upper limb motion assists robot can be denoted as follow

$A_{1} A_{2}=\left[\begin{array}{cccc}\cos \theta_{1} \cos \theta_{2} & -\cos \theta_{1} \sin \theta_{2} & -\sin \theta_{1} & l_{2} \cos \theta_{1} \\ \sin \theta_{1} \cos \theta_{2} & -\sin \theta_{1} \sin \theta_{2} & \cos \theta_{1} & l_{2} \sin \theta_{1} \\ -\sin \theta_{2} & -\cos \theta_{2} & 0 & l_{1} \\ 0 & 0 & 0 & 1\end{array}\right]=\left[\begin{array}{cccc}N_{x} & O_{x} & A_{x} & P_{x} \\ N_{y} & O_{y} & A_{y} & P_{y} \\ N_{z} & O_{z} & A_{z} & P_{z} \\ 0 & 0 & 0 & 1\end{array}\right]$

From equations (8), the elements of the matrices representing the forward kinematics of the proposed wearable motion assist robot can be rewritten as,

$$
\begin{aligned}
& N_{x}=\cos \theta_{1} \cos \theta_{2} \\
& N_{y}=\sin \theta_{1} \cos \theta_{2}, \\
& N_{z}=-\sin \theta_{2} \\
& O_{x}=-\cos \theta_{1} \sin \theta_{2} \\
& O_{y}=-\sin \theta_{1} \sin \theta_{2}, \\
& O_{z}=-\cos \theta_{2} \\
& A_{x}=-\sin \theta_{1} \\
& A_{y}=\cos \theta_{1}, \\
& A_{z}=0 \\
& P_{x}=l_{2} \cos \theta_{1} \\
& P_{y}=l_{2} \sin \theta_{1}, \\
& P_{z}=l_{1}
\end{aligned}
$$

Inverse kinematics analysis aims to determine the respective angular displacement of each robot joint so that the end effector reaches the desired position and orientation. The angular displacement for the elbow flexion/ extension, $\theta_{I}$ can be obtained from $\mathrm{P}_{\mathrm{x}}$ in Equation (9)-(12), where

$$
\theta_{1}=\cos ^{-1}\left(\frac{P_{x}}{l_{2}}\right)
$$

From Equations (5) and (6), the angular displacement of the motor for forearm pronation/supination can be calculated as

$\theta_{2}=\cos ^{-1}\left(\frac{N_{x} l_{2}}{P_{x}}\right)$,

Therefore, provided that the required position and orientation of the end effector of the wearable upper limb motion assist robot is known, the amount that each motor needs to rotate can be computed using Equations (13) and (14). 


\section{RESULTS AND DISCUSSION}

In the experimental test, the elbow motor has extended up to $70^{\circ}$ to reach the food from the home position. Next, the forearm motor has pronated up to $60^{\circ}$, as depicted in Figure 5, and then it has supinated to scoop the food using the spoon, as illustrated in Figure 6. After that, the elbow motor performs flexion, and the spoon reaches the mouth, as shown in Figure 7. Lastly, the person needs to bend his head a little to eat the food.

Figure 8 denotes the position trajectory of the elbow and forearm motors in the eating activity. The position trajectory of the elbow increases linearly from $0^{0}$ to $70^{\circ}$. It stops while the angular displacement of the forearm rises from $60^{\circ}$ to $120^{\circ}$, causing the pronation of the forearm, and then it supinates back linearly from $120^{\circ}$ to $60^{\circ}$. Then, the motor flexes the elbow to $0^{0}$. The movement of the motor is in line with the operational sequence mentioned in the previous section. This movement is acceptable since both the elbow and forearm motions do not need to be performed simultaneously. In the actual scenario, most of human elbow and forearm move separately and in sequence during eating. The elbow and forearm movements in this arrangement also allow for a more stable and more straightforward motion of the robot in assisting the human arm in carrying food to the mouth. Figure 9 gives the angular position of the elbow joint captured by the potentiometer compared to the desired position. From the figure, there is an error between the desired motion provided by the Arduino command window and the potentiometer reading. This error is still acceptable since the robot elbow joint always follows the desired trajectory with a maximum error of $5^{\circ}$, which is due to the mechanical flaws in the mechanism, such as friction. At this stage of the study, only the elbow joint is equipped with a potentiometer to simplify the mechanical design of the distal section of the upper limb. In this experiment, the robot has been set to complete each eating cycle within 6 seconds. However, this duration may be adjusted depending on the user's disability level and needs.

From the experiment, it has been found that it is necessary to give some clearance between the mouth and the spoon during the final feeding stage to avoid the robot from hurting the user. A 3 seconds delay needs to be programmed onto the robot before it starts the next cycle to ensure that the food has already been transferred to the person's mouth. In the future, this feature may be improved by using a camera so that the position and delay can be automatically adjusted according to the time needed by each user, since the disability level of the users may be different. The disabled with lower functional ability level may require a longer time to transfer the food and vice versa. The automatically adjusted position and delay using the camera will be more accommodating and provides them with comfort in using the wearable motion assist robot.

The success rate of the upper limb motion assist robot for eating task is $80 \%$. The success rate has been calculated based on the equation.

success rate $=\frac{\text { number of successful trials }}{\text { total number of trials }} \times 100$,

From the ten trials conducted, the robot has completed the eating cycle eight times. The robot is light in weight, portable, low-cost, and straightforward. The experiment test had also shown that the selected motor had successfully provided the necessary motor torque in eating. However, from the users' feedback, it is learned that that the robot may not be comfortable to some of them since the linkage's length may not be suitable for their arm size. It may be improved by replacing the current mechanical linkages with the ones with adjustable length. The centre of rotation for the robot joints needs to coincide with the centre of rotation of the user's elbow joints for a smooth and easy movement. Even though the proposed system has successfully assisted the upper limb in realizing eating function, the robot may be further improved by incorporating a higher degree of freedom (DOF) movements for a more flexible and natural human upper limb motion assist for eating. The ergonomic aspects of the robot may be enhanced in the future version of the robot by considering the factors that may increase users' comfortability and adaptability while using the robot. Psychological aspects of the robot design may be studied further to improve the patients' trust and engagement in the robotic rehabilitation process. Some of the methods that can be implemented include adapting the therapy intensities to the patients' psychological states so that they will not be too stressed or bored in conducting the exercises. A graphical interface with natural human dialogues may also be incorporated to motivate the patients to perform all the rehabilitation training.

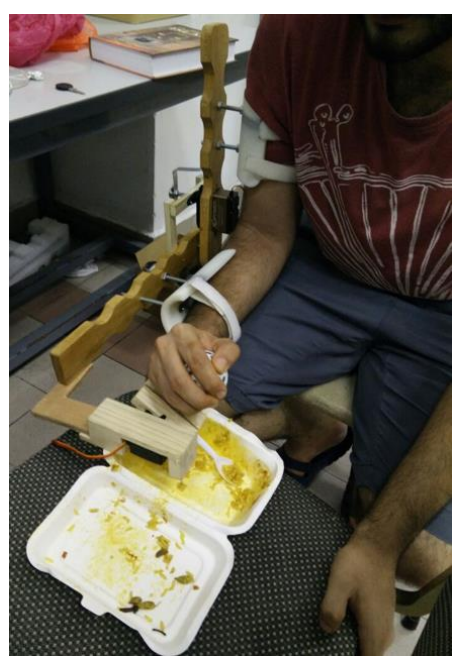

Figure 5. Forearm pronation with the robot's assistance for eating. 


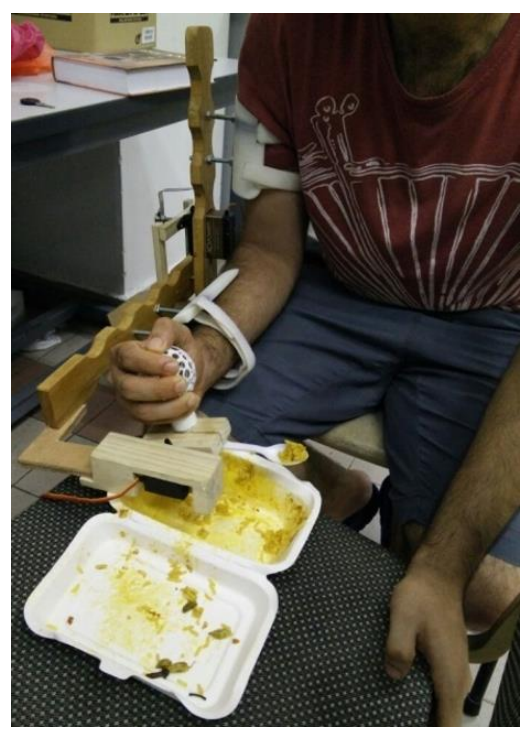

Figure 6. Forearm supination with the robot's assistance for eating

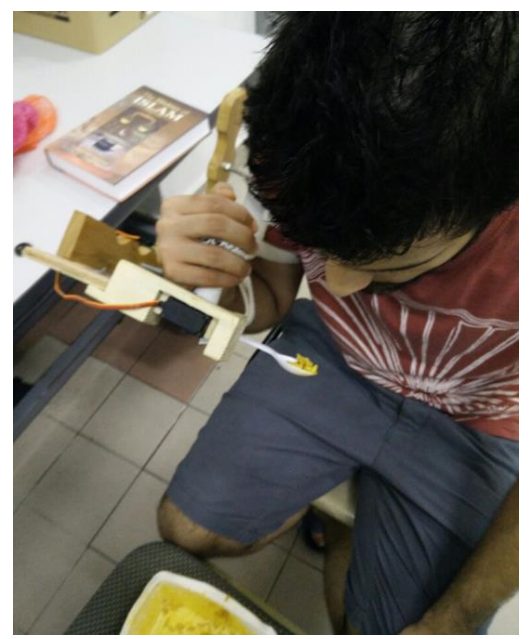

Figure 7. Elbow flexion with the robot's assistance for eating.

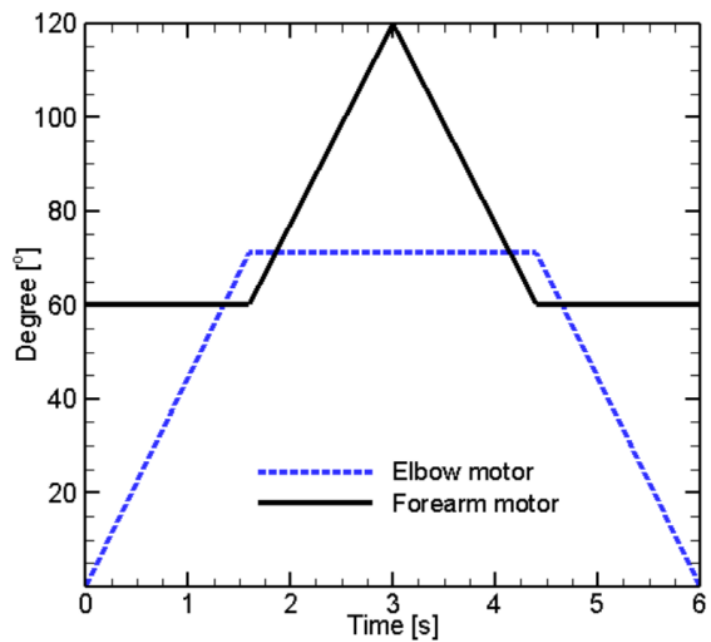

Figure 8. The angular displacement of the elbow and forearm motors in one cycle of eating activity.

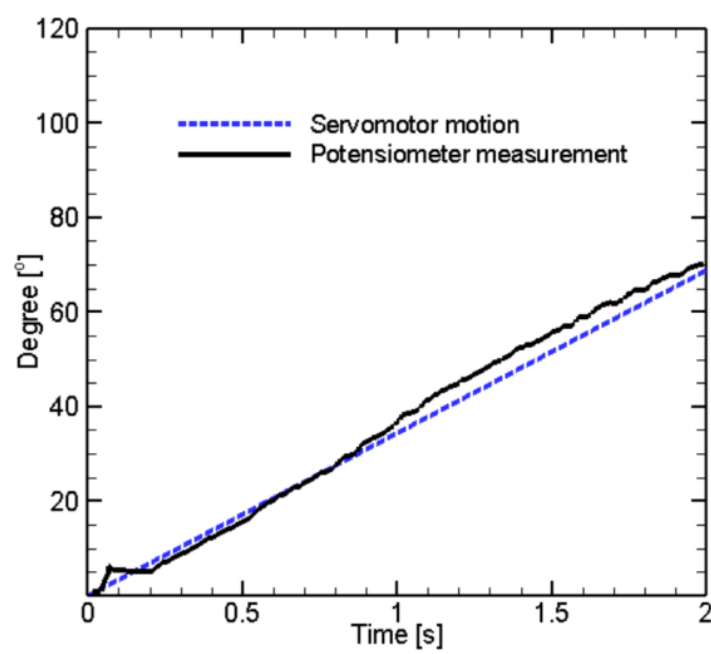

Figure 9. Elbow motor angular displacement during extension.

\section{CONCLUSION}

A wearable upper-limb motion assists robots for helping disabled people to eat by themselves has been presented in this paper. The movement support robot is light-weight, portable, low cost, compact, and straightforward. It is made of two links and two motors actuating the elbow flexion/ extension and forearm pronation/supination movements in eating. The kinematics analysis of the robot has been presented, and the upper limb trajectory in accomplishing the eating task has been set up. The experimental results have revealed that the proposed system was successful in assisting the user in realizing upper limb motions during eating. The wearable robot could also be used for upper limb rehabilitation training purpose in the task-specific eating activity. For future works, the mechanism will be improved with adjustable-length linkages to accommodate various arm sizes. The wooden structure will be replaced with aluminium to increase its durability. The number of DOF may be increased to allow higher flexibility and realize a more natural human arm motion during eating. The robotic mechanism also may be extended for the fingers, wrist, and shoulder movements. More feedback from the users will be collected to improvise the design so that the disabled will be able to wear the motion assist robot by themselves. A camera will be incorporated to obtain the mouth and food position, and the necessary joint angles can be calculated from the vision data. The future study also involves the development of an Assist as Needed (AAN) control law for the system to automatically regulate its assistance level according to the disabled's functional capability level and to encourage their participation in performing the task. 


\section{ACKNOWLEDGEMENT}

The authors would like to thank the International Islamic University Malaysia for supporting this research under the research grant number: P-RIGS18-019-0019.

\section{REFERENCES}

[1] C. C. Fai, Call for better emergency stroke treatment, News Straits Times, 1 May 2019, https:/www.nst.com.my/opinion/letters/2019/05/4 84448/call-better-emergency-stroke-treatment

[2] N. A. Lannin, A. Cusick, A. McCluskey, and R. D. Herbert, Effects of splinting on wrist contracture after stroke: A randomized control trial, Stroke, vol. 38 (issue 1), pp. 111-116, 2007.

[3] J. Difiori, D. Caine, and R. Malina, Wrist pain, distal radial physical injury, and ulnar variance in the young gymnast, The American of Journal Sports Medicine, vol. 34 (issue 5), pp. 840-849, 2006.

[4] Z. Hussain, N. Z. Azlan, and A. Z. Yusof, Human Hand Motion Analysis during Different Eating Activities, Applied Bionics and Biomechanics, pp. 1 12, 2018.

[5] Z. Hussain and N. Z. Azlan, KANE's Method for Dynamic Modeling, Proc. IEEE International Conference on Automatic Control and Intelligent Systems (I2CACIS), Selangor, 174-179, 2016.

[6] K. Kiguchi and R. Matsuo, Accident Prediction based on Motion Data for Perception-Assist with a PowerAssist Robot, Proc. 2017 IEEE Symposium Series on Computational Intelligence (SSCI), Honolulu, Hawaii, 1-5, 2017.

[7] D. Imoto, N. Itoh, S. Kubo, M. Yamaguchi, N. Shimizu, K. Seo et al., Motion analysis of operating a balance exercise assist robot system during forward and backward movements, Journal of Physical Therapy Science, vol. 31 (issue 6), pp. 475-481, 2019.

[8] X. Zhang, T. Luo, A. Tsukahara, and M. Hashimoto, A Coordination Control for a Wearable Walking-Assist Robot, Proc. IEEE International Conference on Robotics and Biomimetics (ROBIO), Macau, 1648-1653, 2017.

[9] Y. P. Sun, S. C. Chen, Y. C. Liang and L. N. Wu, Design of a bionic-inspired exoskeleton robot for lower limb assist, Journal of Vibroengineering, vol. 18 (issue 8), pp. 5452-5461, 2016.
[10] H. J. Lee, S. Lee, W. H. Chang, K. Seo, Y. Shim. B. O. Choi et al., A wearable hip assists robot can improve gait function and cardiopulmonary metabolic efficiency in elderly adults, IEEE Transactions on Neural Systems and Rehabilitation Engineering, vol. 25 (issue 9), pp. 1549-1557, 2017.

[11] S. Masroor, H. Bulut, Bahrudin, and C. Y. Lin, Powered Mobility and Meal Preparing Assistive Devices for Physically Disabled Persons Proc. IFToMM International Symposium on Robotics and Mechatronics ISRM 2019: Robotics and Mechatronics, Taipei, 16-28, 2019.

[12] D. Park, Y. Hoshi, H. P. Mahajan, H. K. Kim, Z. Erickson, W. A. Rogers et al., Active robot-assisted feeding with a general-purpose mobile manipulator: Design, evaluation, and lessons learned, Robotics and Autonomous Systems, vol. 124, pp. 1-18, 2020.

[13] M. Guo, P. Shi, and H. Yu, Development a Feeding Assistive Robot for Eating Assist, Proc. 2nd AsiaPacific Conference on Intelligent Robot Systems (ACIRS), Wuhan, 299-304, 2017.

[14] T. Nozaki, T. Murakami, T. Shimono, K. Ohnishi and R. Oboe, Development of Meal Assistance Device for Patients with Spinal Cord Injury, Proc. IEEE 14th International Workshop on Advanced Motion Control (AMC), Auckland, 388-393, 2016.

[15] D. Yoshioka, M., Ding, G. A. G. Ricardez, J. Takamatsu, and T. Ogasawara, Scoop the SemiLiquid Objects Using a Spoon-Equipped Robot Arm for Meal Support, Proc. ASME 2018 Dynamic Systems and Control Conference, Georgia, USA, 1-9, 2018.

[16] T. Higuma, S. Nakashima, K. Tanaka, and S. Mu, Meal Assistant Robot with Omnidirectional Mobile Plate, Proc. 7th ACIS International Conference on Applied Computing and Information Technology, Honolulu, USA 1-6, 2019.

[17] T. Rhodes and M. Veloso, Robot-driven Trajectory Improvement for Feeding Tasks, Proc. IEEE/RSJ International Conference on Intelligent Robots and Systems (IROS), Madrid, 2991-2996, 2018.

[18] H. Tomimoto, S. Aramaki, S. Nakashima, S. Mu, K. Haruyama and K. Tanaka, Meal-Assistance Robot Operated by Head Movement, Proc. International Conference on Applied Computing and Information Technology ACIT 2017: Applied Computing \& Information Technology, Hamamatsu, Japan, 1-12, 2017. 
[19] H. Tomimotoa, M. Kirihara, S. Mu, S. Nakashima, K. Tanaka, and K. Haruyama, Meal Assistance Robot with Blink Operation Interface, International Conference on Industrial Application Engineering, Matsue, Japan, 297-302, 2016.

[20] C. J. Perera, T. D. Lalitharatne and K. Kiguchi, EEG-Controlled Meal Assistance Robot with Camerabased Automatic Mouth Position Tracking and Mouth Open Detection, Proc. IEEE International Conference on Robotics and Automation (ICRA), Singapore, 1760-1766, 2017.

[21] X. Zhang, X. Wang, B. Wang, T. Sugi, and M. Nakamura, Meal assistance system operated by electromyogram (EMG) signals: Movement onset detection with adaptive threshold, International Journal of Control, Automation and Systems vol. 8, pp. 392-397, 2010.

[22] S. Y. Lee, T. H. Kang, D. B. Shin, D. J. Kim, S. H. Eom and J. Moon, Upper Limb Rehabilitation Robot for Meal Assistance or Meal Rehabilitation Training and Method, US9662525B2 (Patent), 2013.

[23] H. Inoue and T. Noritsugu, Development of upperlimb power-assist machine using linkage mechanism, Journal of Robotics and Mechatronics, vol. 30 (issue 2), pp. 214-222, 2018

[24] K. Kiguchi, M. H. Rahman, M. Sasaki, and K. Teramoto, Development of a 3DOF mobile exoskeleton robot for human upper-limb motion assist, Robotics and Autonomous Systems, vol. 56 (issue 8), pp. 678-691, 2008.

[25] K. Kiguchi, R. A. R. C. Gopura, and Y. Hayashi, The Effect of Impedance Parameters in 7DOF Upper-Limb Power-Assist Exoskeleton Robot, Proc. First IFToMM Asian Conference on Mechanism and Machine Science, Taipei, Taiwan, 21 - 25, 2010.
[26] H. Kozuka, D. Ushijima and H. Tachiya, Motionassist arm with a passive joint for an upper limb, Journal of Robotics and Mechatronics, vol. 32 (issue 1), pp. 183-198, 2020.

[27] R. F. Natividad, S. W. Hong, T. M. Jackson, and C. H. Yeow, The Exosleeve: A Soft Robotic Exoskeleton for Assisting in Activities of Daily Living, Wearable Robotics: Challenges and Trends. Biosystems \& Biorobotics, vol. 22, pp. 406409, 2018.

[28] L. T. Hu, C. L. Cin, X. D. Wu, D. S. Zhao, D. H. Ye, B. Shu, et al., An Upper Limb Motion Assist Device for Hemiplegic Patients, Proc. IEEE International Conference on Cyborg and Bionic Systems (CBS), Shenzhen, China, 199-203, 2018.

[29] Y. Liao, C. Zong, H. Lee, and E. Tanaka, Development of Kinect-based Upper-Limb Assistance Device for the Motions of Activities of Daily Living, Proc. IEEE International Conference on Cyborg and Bionic Systems (CBS), Shenzhen, China, 222-227, 2018.

[30] B. Brahmi, M. Saad, C. O. Luna and P. S. Archambaul, Passive and active rehabilitation control of human upper-limb exoskeleton robot with dynamic uncertainties, Robotica, vol. 36 (issue 11), pp. 1757-1779, 2018

[31] A. M. Khan, D. W. Yun, K. M. Zuhaib and J. Iqbal, Estimation of desired motion intention and compliance control for upper-limb assist exoskeleton, International Journal of Control, Automation and Systems, vol. 15, pp. 802-814, 2017. 